\section{Advances in Mass Spectrometry,} Volume 12 Proceedings of the 12th International Mass Spectrometry Conference Held in Amsterdam, August 26-30, 1991

Edited by P. G. Kistemaker and N. M. M. Nibbering Elsevier Science Publishers, B, V., Amsterdam. xxii + 951 pp., ISBN \#0-444-88871-3, 1992, US\$281.50, Dfl. 450.00

Book review by Charles L. Wilkins Department of Chemistry University of California, Riverside Riverside, CA 92521

This volume is a compilation of the manuscripts of most of the plenary and invited keynote lectures presented at the 12th triennial conference on mass spectrometry. The papers contained herein are reprinted from Volumes 118 and 119 of the International Journal of Mass Spectrometry and Ion Processes. Because the aim of the conference was to "... cover all aspects of mass spectrometry including theory, fundamental studies, applications and instrumentation...", with particular emphasis on developments during the three years since the last conference, it is not surprising that the 32 review papers presented here average almost 30 pages each. As usual in an edited volume, particularly one of this nature, there is some variation in the quality and depth of individual contributions.

A reasonable criterion for judging any review article is the depth of its discussion and the comprehensiveness of its literature coverage. Although this volume contains a few superficial and narrow papers, most are of high quality and satisfy reasonable expectations for the volume. A few of the papers that are especially outstanding are the contributions by March, Baillie, Speranza, Hieftje and Norman, and Gelpi.

March contributes an excellent discussion of ion trap mass spectrometry, documented with almost 300 references. Ballie discusses advances in applications of mass spectrometry to studies of drug metabolism, pharmacokinetics, and toxicology, citing over 200 articles, primarily from the 1989-1991 period, and Gelpi, in a related article, reviews recent trends in biochemical and biomedical applications of mass spectrometry. This latter article contains an interesting bibliometric cvaluation of the MEDLINE database for the period 1988-1991, where 4000 entries are found for mass spectrometry. An example of the type of finding which results is the observation that, by far, most of the uses of mass spectrometry in human studies were reported in the Journal of Chromatography (169 papers), almost double the number appearing in the runner-up Biomedical and Environmental Mass Spectrometry (89 papers). Speranza presents an excellent discussion of gas-phase ion chemistry versus solution chemistry in a well written article with about 100 current literature references. Finally, Hieftje and Norman review plasma source mass spectrometry, including a comprehensive literature review (with titles and organized by sub-topics) containing 492 references.

The high standard set by the authors mentioned above is met to a large degree, albeit less comprehensively, by most of the other contributors. The few exceptions, which will be left to the reader to identify, are sufficiently short and limited in number that they do not detract significantly from the comprehensive reviews of the entire field of mass spectrometry, as intended. The quality of the production is exceptional and the book is remarkably error free. Anyone seeking an overview of advances in mass spectrometry during the 1988-1991 period would do well to start here. There is no doubt that every science library should have a copy of the present volume. Whether the cost of the volume justifies inclusion in a personal library is less clear. Incidentally, if you wonder which of the invited speakers did not see fit to contribute manuscripts, they are listed on p. 875, immediately preceding the listings of poster session papers, titles, and authors, which takes up the next 58 pages of the book. A comprehensive author index follows. However, there is no subject index. 